\title{
ESTABILIDAD DE RENDIMIENTOS DE 5 GENOTIPOS DE GYNERIUM SAGGITATUM AUBL., BAJO DOS NIVELES DE FERTILIZACIÓN EN DIEZ LOCALIDADES DEL DEPARTAMENTO DE CÓRDOBA, COLOMBIA
}

\author{
STABILITY OF YIELDS OF 5 GENOTYPES OF GYNERIUM \\ SAGGITATUM AUBL., UNDER TWO LEVELS OF FERTILIZATION \\ IN TEN LOCALITIES OF THE DEPARTMENT OF CÓRDOBA, COLOMBIA
}

\author{
Jaime Hernández Burgos ${ }^{1}$ \\ Alfredo Jarma Orozco ${ }^{2}$ \\ Alicia Humanez Álvarez 3 \\ Eder Durango Ballesteros ${ }^{4}$ \\ ${ }^{1}$ Ingeniero Agrónomo, Magister en Ciencias Agronómicas, \\ Universidad Nacional Abierta y a Distancia - CCAV, Sahagún-Córdoba, (Colombia). \\ 2 Ingeniero Agrónomo, Doctor en Ciencias Agrarias, \\ Universidad de Córdoba, Facultad de Ciencias Agrícolas Montería Córdoba. \\ ${ }^{3}$ Bióloga, Doctora en Biología vegetal y Edafología. \\ Universidad del Sinú "Elías Bechara Zainum", Facultad de Ciencias e ingenierías, Departamento \\ de Ciencias Básicas, Laboratorio de Investigaciones Biológicas, Montería (Colombia). \\ ${ }^{4}$ Ingeniero Agrónomo, Doctor en Biotecnología Agrícola. \\ SENA. Centro Agroempresarial y Minero, SENNOVA. Cartagena (Colombia). \\ 1 jaime.hernandez@unad.edu.co \\ ${ }^{3}$ aliciahumanez@unisinu.edu.co
}

\begin{abstract}
Resumen
La caña flecha (Gynerium saggittatum Aubl.) se ha convertido en sustento económico y de perpetuación cultural de antiguos pueblos indígenas de la costa norte colombiana. Sin embargo, el desconocimiento del comportamiento de este cultivo en diversas condiciones edafoclimáticas, generan baja capacidad de producción. En el presente trabajo se analizaron los rendimientos de fibra y fotosíntesis de
\end{abstract}

5 genotipos de caña flecha bajo dos niveles de fertilización en diez localidades del departamento de Córdoba: Bajo Grande, La Abisinia, San Martin, San Efraín, Tófeme, Buenos Aires, INTECAVI, Bella Cecilia, Chimalito y La Oportunidad, se utilizó un diseño de bloques completos al azar con arreglo factorial $5 \times 2$ : cinco (5) genotipos (Criolla, Criolla 1, Criolla 2, Martinera y Costanera), sometidos a dos (2) 
niveles de fertilización: con y sin fertilización orgánica, con tres (3) repeticiones. Se midió la longitud de la nervadura central (LNC) y las tasas de intercambio gaseoso fotosíntesis neta, concentración interna de $\mathrm{CO}_{2}$, conductancia estomática y transpiración. Se analizaron los rendimientos de fibra de acuerdo con el método de Lin y Binns (1988) y Carneiro (1998). Se realizó análisis de varianza y pruebas de comparación de medias de Duncan al nivel $\mathrm{a}=0.05$ de acuerdo con el diseño planteado, utilizando el paquete estadístico SAS 9.1. Se evidenciaron mayores tasas de fotosíntesis y rendimientos de fibra en los genotipos Criolla y Criolla 1 con respecto al resto, así como un mejor desarrollo de las plantas en las localidades de Buenos Aires, Bella Cecilia y Chimalito. Se obtuvieron rendimientos de fibra más estables cuando se aplicó fertilización orgánica. Los resultados sugieren fomentar el establecimiento de los genotipos Criolla y Criolla 1 en las localidades de Buenos Aires, Bella Cecilia y Chimalito, bajo aplicación de fertilizantes orgánicos que mejoren su productividad y eviten el agotamiento de los suelos.

Palabras clave: caña flecha, estabilidad fenotípica, fotosíntesis, rendimientos.

\footnotetext{
Abstract

Caña Flecha (Gynerium saggittatum Aubl.) has become an economic and cultural perpetuation of ancient indigenous peoples of the north coast of Colombia. However, the ignorance of the behavior of this crop in diverse edaphoclimatic conditions, generates low production capacity. In the present work, the fiber and
}

photosynthesis yields of 5 arrow cane genotypes under two levels of fertilization in ten localities of the department of Córdoba were analyzed: Bajo Grande, La Abisinia, San Martin, San Efrain, Tófeme, Buenos Aires, INTECAVI, Bella Cecilia, Chimalito and la Oportunidad, it was used a randomized complete block design with $5 \times 2$ factorial arrangement: five (5) genotypes (Criolla, Criolla 1, Criolla 2, Martinera y Costanera), subjected to two (2) fertilization levels without organic fertilization with three (3) replicates. The length of the central rib (LNC) and the rates of gaseous exchange net photosynthesis, internal concentration of $\mathrm{CO}_{2}$, stomatal conductance and transpiration were measured. Fiber yields were analyzed according to the method of Lin y Binns (1988) y Carneiro (1998). Duncan analysis of variance and comparing means at the $a=0.05$ level were made according to the proposed design, using SAS 9.1 statistical package. There were higher rates of photosynthesis and fiber yields in the CrioIla and Criolla 1 genotypes compared to the rest, as well as a better development of the plants in the localities of Buenos Aires, Bella Cecilia and Chimalito. More stable fiber yields were obtained when organic fertilization was applied. The results suggest to encourage the establishment of Criolla and Criolla 1 genotypes in the localities of Buenos Aires, Bella Cecilia and Chimalito, under the application of organic fertilizers that improve his productivity and avoid the depletion of the soils.

Keywords: cana flecha, phenotypic stability, photosynthesis, yield. 


\section{Resumen gráfico}

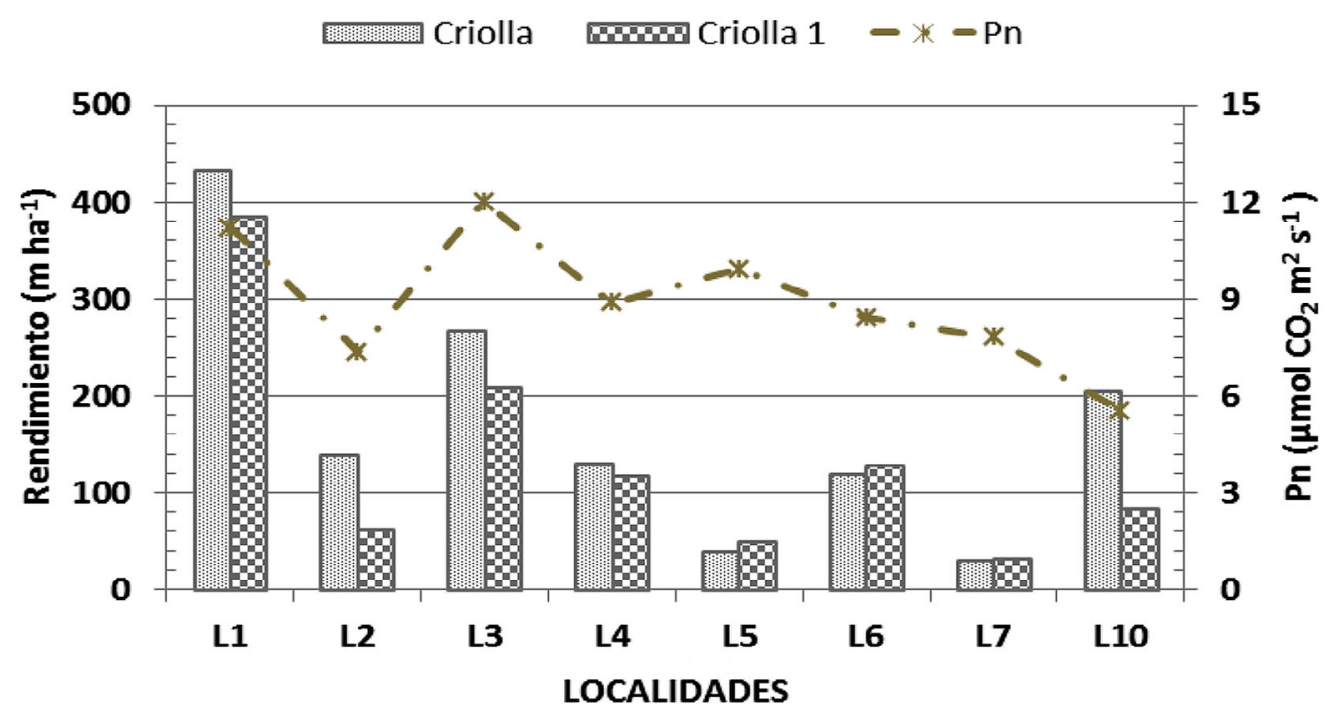

Fotosíntesis y rendimientos (Criolla y Criolla 1 ) de caña flecha en 8 localidades del departamento de Córdoba.

\section{Introducción}

La caña flecha por ser una especie silvestre, originaria del continente americano, crece desde México hasta Paraguay e incluso en las islas del Caribe con excepción de las Bahamas (SNE 2004). En Colombia se encuentra en varios departamentos como: Antioquia, Arauca, Bolívar, Casanare, Cauca, Córdoba, Cundinamarca, Guajira, Huila, Putumayo, Sucre, Tolima y Vichada; sin embargo, solo en Córdoba y Sucre esta planta es usada con fines artesanales. (Casas Caro et al. 2010). En Córdoba, en el resguardo indígena de Tuchín y San Andrés de Sotavento cuenta actualmente con 93 ha y se requieren 145,4 ha adicionales para suplir dicha demanda caña flecha. (López Rojas et al. 2014). La identificación de ecotipos, la producción masiva de materias primas y transformación de las mismas a un costo sostenible y competitivo es fundamental para la conservación de una cultura milenaria.

Desde un punto de vista comercial el principal componente es la nervadura central de la hoja de caña flecha, la cual se usa como materia prima para la elaboración de artesanías representativas de la cultura colombiana, en tanto que el tallo puede ser se empleado para cercar las casas; la espiga de la inflorescencia, para flechas de pescar; la panoja, como elemento ornamental casero, convirtiéndose esta especie en sustento económico y forma de perpetuación de antiguos pueblos indígenas de la costa norte colombiana (Pastrana y Suarez 2009). Sin embargo, por no estar constituido como sistema de cultivo comercial de escala industrial, sino como un cultivo de pequeña escala, su manejo hasta el momento ha sido de forma empírica, con la aplicación de prácticas tradicionales (SNE 2004).

Al igual que cualquier cultivo la caña flecha necesita condiciones agroecológicas óptimas para su desarrollo si se quieren establecer cultivos comerciales que ayuden a las poblaciones indígenas a obtener materias primas 
más económicas y de forma constante. Las características ambientales de una localidad influyen directamente sobre el crecimiento de las plantas y por supuesto en la calidad de los productos (Aramendiz et al. 2005), por lo que es común encontrar en la literatura la relación de la oferta ambiental sobre la tasa de crecimiento y sobre la acumulación de la biomasa (Mekonnen et al. 2006). Las variaciones de la época de siembra, la población y la incidencia de factores ambientales como radiación solar, precipitación, temperatura, etc. pueden afectar las expresiones fenotípicas y fisiológicas de las plantas, ya que se ven afectados procesos como la fotosíntesis, la transpiración, el alargamiento de células, el crecimiento y competencia entre especies (Jarma, 2008).

La carencia de un plan de manejo agronómico, de planes nutricionales, semillas de óptima calidad y el desconocimiento del comportamiento de este cultivo cuando se somete a diversas condiciones edafoclimáticas generan la baja capacidad de producción debido a que no se pueden trazar estrategias que permitan disminuir los tiempos de producción, mejorar los rendimientos y la calidad del producto, entre otros factores (Rivera et al. 2009; Durango y Humanez, 2017). El conocimiento y explicación de la expresión fenotípica de los diferentes genotipos de caña flecha cuando son expuestos a diferentes condiciones agroecológicas son necesarios para conocer y entender aspectos relacionados con las variables de rendimiento y producción. El objetivo de este trabajo fue evaluar el rendimiento y parámetros fisiológicos de cinco (5) genotipos de caña flecha (Gynerium saggittatum Aubl.), en los resguardos indígenas de San Andrés de Sotavento y Tuchín, en el departamento de Córdoba, bajo dos (2) niveles de fertilización.

\section{Material Y Métodos}

Se seleccionaron 10 localidades en los municipios de San Andrés de Sotavento (Bajo Grande, La Abisinia, San Martin, San Efraín, Tófeme) y Tuchín (Buenos Aires, INTECAVI, Bella Cecilia, Chimalito y La Oportunidad) en el departamento de Córdoba, Colombia. En cada localidad se delimitaron lotes de dos (2) hectáreas en la cuales se estableció un diseño de bloques completos al azar con arreglo factorial 5x2: cinco (5) genotipos (Criolla, Criolla 1, Criolla 2, Martinera y Costanera), sometidos a dos (2) niveles de fertilización (con y sin fertilización orgánica) con tres (3) repeticiones, para un total de 30 unidades experimentales por localidad. Cada unidad experimental estuvo constituida por 10 plantas y para efectos de las mediciones se utilizaron 6 plantas por unidad experimental. Todas las plantas se encontraban en similares condiciones fisiológicas y en óptimas condiciones fitosanitarias.

Como variable de rendimiento $(\mathrm{m})$ se consideró la longitud de la nervadura central (LNC), por estado nutricional, genotipo y localidad. En el período de máximo crecimiento (fase exponencial), en cada tratamiento se midieron las tasas de intercambio gaseoso con un medidor de gases al infrarrojo (IRGA). Se determinó la tasa de fotosíntesis neta $\mathrm{Pn}\left(\mu \mathrm{mol} \mathrm{CO}_{2}\right.$ $\left.\mathrm{m}^{-2} \mathrm{~s}^{-1}\right)$, concentración interna de $\mathrm{CO}_{2} \mathrm{Ci}(\mathrm{ppm})$, conductancia estomática gs $\left(\mathrm{mmol} \mathrm{CO}_{2} \mathrm{~m}^{2} \mathrm{~s}^{-1}\right)$, transpiración $\mathrm{E}\left(\mathrm{mmol} \mathrm{H}_{2} \mathrm{O} \mathrm{m}^{-2} \mathrm{~s}^{-1}\right)$. Para homogenizar las lecturas entre genotipos, se utilizó una radiación fotosintéticamente activa (PAR) estable de $1200 \mu \mathrm{mol}$ de fotones $\mathrm{m}^{-2} \mathrm{~s}^{-1} \mathrm{y}$ una concentración fija de $\mathrm{CO}_{2}$ de 380 ppm.

Se aplicaron parámetros de estabilidad fenotípica para el rendimiento de fibra de acuerdo al método de Lin y Binns (1988), en el cual el desempeño general de los genotipos se define como el cuadrado medio de la distancia 
entre la media del cultivar y la respuesta media máxima para todas las localidades, de tal modo que los genotipos, localidades y niveles de nutrición con menores valores corresponden a los de mayor estabilidad. Posteriormente se obtuvo el estadístico Pig de acuerdo con Carneiro (1998), para determinar genotipos superiores en ambientes favorables y desfavorables. Los modelos son los siguientes:

\section{Método de Lin y Binns (1988).}

Definido como el cuadrado medio de la distancia entre la media del nivel de tratamiento y la respuesta media máxima para todas las localidades de tal modo, que la localidad, genotipo o nivel de fertilización con menores valores corresponde a los de mayor estabilidad. De acuerdo con esta definición el estimador Pig es determinado por la siguiente fórmula:

$P_{i g}=\frac{\sum_{j=1}^{n}\left(X_{i j}-M_{j}\right)^{2}}{2 n} \quad($ Ecuación 1)

Donde:

Pig = estadístico del parámetro de estabilidad de la localidad, genotipo o nivel de fetilización i;

$\boldsymbol{X} \boldsymbol{i} \boldsymbol{j}=$ respuesta de la variable dependiente del i - ésima localidad, genotipo o nivel de fertilización en la j - ésima localidad;

$\boldsymbol{M j}$ = respuesta máxima observada entre todas las localidades, genotipo o nivel de fertilización en la localidad j;

$\boldsymbol{n}=$ número de localidades.

\section{Método Carneiro (1998).}

Descompone el estadístico Pig, con el fin de identificar los genotipos superiores en ambientes favorables y desfavorables, utilizando la misma metodología de clasificación de ambientes de Eberhart y Russell (1966). En esta descomposición se estimaron los estadísticos siguientes:

$$
P_{i f}=\frac{\sum_{j=1}^{n}\left(x_{i j}-M_{j}\right)^{2}}{2 f} \text { y } P_{i d}=\frac{\sum_{j=1}^{n}\left(x_{i j}-M_{j}\right)^{2}}{2 \mathrm{~d}}
$$

(Ecuación 2)

Dónde:

Pif = estadístico de Pig para localidades o niveles de fertilización favorables;

Pid = estadístico de Pig para localidades o niveles de fertilización favorables desfavorables;

$\boldsymbol{f}=$ número de localidades o niveles de fertilización desfavorables;

$\boldsymbol{d}=$ número de localidades o niveles de fertilización desfavorables;

$\boldsymbol{X} \boldsymbol{i j}$ y $\mathbf{M} \boldsymbol{j}$ como fueron definidos anteriormente.

Para las variables de intercambio gaseoso y de crecimiento y desarrollo, en cada una de las localidades se realizó una prueba de normalidad de Shapiro Wilk, prueba de homogeneidad de varianza, análisis de varianza y pruebas de comparación de medias de Duncan al nivel $a=0.05$ de acuerdo al diseño planteado combinado con el paquete estadístico SAS 9.1.

\section{Resultados Y Discusión}

\section{Análisis de varianza $y$ estabilidad}

En la tabla 1 , se pueden observar los rendimientos promedios de nervadura para cada genotipo y localidades tanto en ambientes favorables como desfavorables. Los valores de rendimientos oscilaron entre $0,1 \mathrm{~m}$ para la localidad de San Martin y 289,4 m para Buenos Aires. Cuando se discrimina entre ambientes 
favorables y desfavorables, se observa que los rendimientos estuvieron entre $120 \mathrm{~m}$ y 289,2 para localidades favorables y entre $0,1 \mathrm{~m}$ y $88,9 \mathrm{~m}$ para las localidades clasificadas como desfavorables. Para el caso de tratamientos:
Criolla F $(138,5)$ Criolla NF $(138,5)$ y Criolla $1 \mathrm{~F}$ $(128,2)$ presentaron los mejores rendimientos y estabilidad tanto en localidades favorables como desfavorables (tabla 2).

Tabla 1. Rendimiento de fibra $(m)$ de 5 genotipos de caña flecha evaluados en 10 localidades y dos niveles de nutrición y clasificación de localidades favorables y desfavorables.

\begin{tabular}{|c|c|c|c|c|c|c|c|c|c|c|c|}
\hline \multirow{2}{*}{ GENOTIPOS } & \multicolumn{10}{|c|}{ LOCALIDADES } & \multirow{2}{*}{$\begin{array}{l}\text { PROMEDIO } \\
\text { TRATAMIENTO }\end{array}$} \\
\hline & L1 & L2 & L3 & L4 & $\mathbf{L 5}$ & L6 & L7 & L8 & L9 & L10 & \\
\hline CRIOLLA NF & 477,3 & 150,9 & 262,5 & 184,7 & 25,8 & 133,8 & 39,2 & 24,1 & 0,0 & 87,2 & 138,5 \\
\hline CRIOLLA F & 386,9 & 126,0 & 269,0 & 72,1 & 50,2 & 103,7 & 18,8 & 52,9 & 0,0 & 320,9 & 140,0 \\
\hline CRIOLLA 1 NF & 246,3 & 71,2 & 191,8 & 124,4 & 35,1 & 54,2 & 36,3 & 104,1 & 0,0 & 74,1 & 93,7 \\
\hline CRIOLLA 1 F & 520,4 & 50,8 & 222,6 & 110,4 & 62,1 & 199,9 & 24,2 & 0,0 & 0,3 & 91,3 & 128,2 \\
\hline CRIOLLA 2 NF & 185,0 & 113,6 & 175,8 & 124,9 & 31,5 & 40,5 & 20,7 & 45,1 & 0,0 & 49,2 & 78,6 \\
\hline CRIOLLA 2 F & 221,5 & 110,1 & 193,9 & 112,3 & 34,3 & 121,2 & 30,9 & 0,0 & 0,0 & 133,0 & 95,7 \\
\hline MARTINERA NF & 254,5 & 73,0 & 91,6 & 131,3 & 67,7 & 50,9 & 33,1 & 17,8 & 0,0 & 43,0 & 76,3 \\
\hline MARTINERA F & 228,6 & 35,6 & 157,9 & 88,8 & 62,6 & 33,3 & 26,8 & 33,3 & 0,0 & 66,1 & 73,3 \\
\hline COSTANERA NF & 145,4 & 16,2 & 144,9 & 157,9 & 176,7 & 71,7 & 48,3 & 60,0 & 0,8 & 50,3 & 87,2 \\
\hline COSTANERA F & 228,6 & 44,4 & 183,0 & 93,2 & 29,2 & 80,0 & 72,4 & 0,0 & 0,0 & 44,8 & 77,6 \\
\hline $\begin{array}{l}\text { PROMEDIO } \\
\text { LOCALIDAD }\end{array}$ & 289,4 & 79,2 & 189,3 & 120,0 & 57,5 & 88,9 & 35,1 & 33,7 & 0,1 & 96,0 & 98,9 \\
\hline \multirow[t]{2}{*}{ Diferencia } & 190,5 & $-19,7$ & 90,4 & 21,1 & $-41,4$ & $-10,0$ & $-63,8$ & $-65,2$ & $-98,8$ & $-2,9$ & \\
\hline & $f$ & d & $\mathrm{f}$ & $f$ & d & d & d & d & d & d & \\
\hline
\end{tabular}

L1: Buenos Aires, L2: INTECAVI, L3: Bella Cecilia, L4: Chimalito, L5: La Oportunidad, L6: Bajo Grande, L7: La Abisinia, L8: San Martin, L9: San Efraín, L10: Tófeme. f: localidad favorable, d: localidad desfavorable. Los términos NF y $\mathrm{F}$ que aparecen al lado de cada genotipo corresponden a No Fertilizado y Fertilizado respectivamente.

La tabla 2, muestra que, para el caso de los tratamientos, combina el efecto del genotipo y la nutrición, los genotipos Criolla y Criolla 1 con y sin fertilización tuvieron los menores parámetros Pig, indicando que el rendimiento de fibra de estos genotipos muestra mayor estabilidad tanto cuando se fertiliza como al no fertilizarse. De igual forma sucede si se evalúa solo el factor genotipo, se observa que Criolla y Criolla 1 muestran la mayor estabilidad de los rendimientos de fibra cuando son evaluadas en todas las localidades. Igualmente, la tabla 2 indica que las localidades con menores Pig o más estables para el rendimiento de caña flecha fueron Buenos Aires y Bella Cecilia, ambas ubicadas en el municipio Tuchín. 
Tabla 2. Índices Pig, Pif y Pid para rendimiento de caña flecha de cinco genotipos evaluados a dos niveles de fertilización en 10 localidades.

\begin{tabular}{|c|c|c|c|c|c|c|c|c|}
\hline \multirow{2}{*}{ Tratamiento } & \multicolumn{2}{|c|}{ Pig } & \multicolumn{2}{|c|}{ Pif } & \multicolumn{2}{|c|}{ Pid } & \multirow{2}{*}{ Loc } & \multirow{2}{*}{ Pig Loc } \\
\hline & Tto & Gen & Tto & Gen & Tto & Gen & & \\
\hline Criolla NF & 4557,8 & \multirow{2}{*}{273} & 316,6 & \multirow{2}{*}{$0,0 *$} & 6375,4 & \multirow{2}{*}{390,0} & L1 & 48,9 \\
\hline Criolla F & 3093,7 & & 2970,4 & & 2241,0 & & L2 & 28656,5 \\
\hline Criolla 1 NF & 9726,8 & \multirow{2}{*}{1524,8} & 13510,7 & \multirow{2}{*}{$987,3 *$} & 7845,0 & \multirow{2}{*}{1755,2} & L3 & 9355,1 \\
\hline Criolla 1 F & 4832,8 & & 358,2 & & 6356,6 & & L4 & 21825,8 \\
\hline Criolla 2 NF & 12632,4 & \multirow{2}{*}{4078,9} & 20195,8 & \multirow{2}{*}{9833,4} & 9135,1 & \multirow{2}{*}{1612,7} & L5 & 36632,1 \\
\hline Criolla 2 F & 8809,0 & & 15825,2 & & 5428,1 & & L6 & 24069,3 \\
\hline Martinera NF & 11566,5 & \multirow{2}{*}{4810,2} & 17018,0 & \multirow{2}{*}{9416,2} & 9026,3 & \multirow{2}{*}{2836,2} & L7 & 40577,8 \\
\hline Martinera F & 11637,7 & & 16243,9 & & 9006,1 & & L8 & 41826,3 \\
\hline Costanera NF & 13351,1 & \multirow{2}{*}{5486,1} & 25999,5 & \multirow{2}{*}{11738,3} & 7879,2 & \multirow{2}{*}{2806,6} & L9 & 49602,1 \\
\hline Costanera F & 11771,3 & & 15420,5 & & 9609,6 & & L10 & 25869,5 \\
\hline \multicolumn{2}{|l|}{ Fertilización } & \multicolumn{2}{|c|}{ Pig } & \multicolumn{2}{|r|}{ Pif } & \multicolumn{3}{|c|}{ Pid } \\
\hline \multicolumn{2}{|l|}{ NF } & \multicolumn{2}{|c|}{523,27} & \multicolumn{2}{|r|}{683,41} & & \multicolumn{2}{|c|}{454,64} \\
\hline \multicolumn{2}{|l|}{$\mathbf{F}$} & \multicolumn{2}{|c|}{$201,82 *$} & \multicolumn{2}{|r|}{$404,28 *$} & & \multicolumn{2}{|c|}{$115,05^{*}$} \\
\hline
\end{tabular}

L1: Buenos Aires, L2: INTECAVI, L3: Bella Cecilia, L4: Chimalito, L5: La Oportunidad, L6: Bajo Grande, L7: La Abisinia, L8: San Martin, L9: San Efraín, L10: Tófeme, NF: No fertilizado, F: Fertilizado.

Igualmente, se realizó la descomposición del parámetro de estabilidad aplicando el modelo de Carneiro, el cual descompone el comportamiento de los tratamientos teniendo en cuenta si están en una localidad favorable o desfavorable (tabla 3). Para el caso de las localidades que se clasificaron como favorables en la tabla 1 (Buenos Aires, Bella Cecilia y Chimalito), se puede observar que los genotipos Criolla y Criolla 1 con y sin fertilizar presentan los menores indicadores de estabilidad Pif. De igual forma, en el caso de las localidades desfavorables (INTECAVI, La Oportunidad, Bajo Grande, La Abisinia, San Martin, San Efraín y Tófeme), la tabla 2 muestra que los genotipos Criolla y Criolla 1 con y sin fertilizar presentan los menores indicadores de estabilidad Pid, indicando así esta prueba, que los genotipos más estables tanto en condiciones favorables como desfavorables son Criolla y Criolla 1.

Estos resultados muestran que los genotipos se comportan diferencialmente en diversos ambientes, esto es lo que se denomina interacción genotipo por ambiente (Souza 2005) y esta representa una de las principales dificultades para obtener rendimientos altos y estables en los procesos de selección, tanto de genotipos como de ambientes naturales o inducidos para obtener los mejores rendimientos (Pérez et al. 2005)

La tabla 2 también muestra los Índices de estabilidad del rendimiento de fibra, para ambientes favorables (Pif) y desfavorables (Pid) de los cinco (5) genotipos de caña flecha cuando son sometidos a dos niveles de 
fertilización en las 10 localidades, indicando que el nivel de fertilización orgánica mostró menores Pig, Pif y Pid, indicando que se obtienen rendimientos de fibra más estables cuando se realiza fertilización ya sea en localidades favorables o desfavorables.

\section{Parámetros fisiológicos}

En la tabla 3, se muestran los resultados de los parámetros fisiológicos. Las mediciones se hicieron en las localidades de Buenos Aires, INTECAVI, Bella Cecilia, Chimalito, La Oportunidad, Bajo Grande, La Abisinia y Tófeme. No se realizó en las localidades de San Martin y San Efraín debido a que el porcentaje de mortalidad en estas localidades estuvo alrededor del $90 \%$ y los resultados no serían confiables y podría conducir a conclusiones erróneas. En el análisis de varianza se detectó un efecto significativo de las localidades en $\mathrm{Pn}, \mathrm{Ci}$, gs y $\mathrm{E}$ sugiriendo un comportamiento diferencial de las regiones sobre la adaptabilidad y eficiencia fisiológica de la especie, coincidiendo con Souza (2005) quien encontró un comportamiento similar en el cultivares de algodón al evaluarlo a diferentes ambientes y con Rodríguez (2011), quien evaluó un grupo de cultivares de trigo en la región de Mexicali en México. También se apreciaron diferencias significativas para fotosíntesis neta en el factor genotipo, indicando que el efecto genético fue más importante que la fertilización.

Al realizar la comparación de medias de Duncan $(0.05 \%)$ se observó que las localidades Buenos Aires y Bella Cecilia presentaron los valores significativamente mayores de Pn $\left(11,2\right.$ y $\left.12,1 \mu \mathrm{mol} \mathrm{CO} \mathrm{m}^{2} \mathrm{~s}^{-1}\right), \mathrm{Ci}(157,8$ y $\left.191,31 \mu \mathrm{mol} \mathrm{CO} \mathrm{m}^{2} \mathrm{~s}^{-1}\right)$, gs $(0,12$ y $0,15 \mu \mathrm{mol}$ $\left.\mathrm{CO}_{2} \mathrm{~m}^{2} \mathrm{~s}^{-1}\right)$ y $\mathrm{E}\left(4,23\right.$ y $\left.4,48 \mu \mathrm{mol} \mathrm{H}_{2} \mathrm{O} \mathrm{m}^{2} \mathrm{~s}^{-1}\right)$ (Figura 1). Es probable que el comportamiento de estas variables en las localidades de Buenos Aires y Bella Cecilia expliquen los resultados obtenidos en el análisis de estabilidad, en el cual estas localidades presentaron los menores índices de Pig, indicando que los rendimientos fueron más estables.

En la tabla 3 también se puede observar el análisis de varianza correspondiente a las variables $\mathrm{Pn}, \mathrm{Ci}$, gs y $\mathrm{E}$ dentro de cada una de las diferentes localidades evaluadas. Se puede apreciar que solo se registraron diferencias estadísticamente significativas para la variable fotosíntesis neta en el factor genotipo en todas las localidades, indicando que el efecto genético fue más importante que la fertilización. Es importante destacar que los valores de Pn observados para caña flecha están muy por debajo de los valores promedios que puede presentar una especie de metabolismo C4 (Taiz \& Zaiger). Los resultados pueden dar una idea aproximada del gran estrés al que estuvieron sometidos los genotipos en todas las localidades, incluso en las dos de mejor comportamiento.

De acuerdo con los resultados anteriores, se puede señalar que el desempeño de un genotipo, depende, fundamentalmente de las condiciones ambientales a las que es sometido (Kang 1998; Ruiz et al.,2002) y la interacción genotipo ambiente obedece, en parte a la ocurrencia de algún tipo de estrés (Carbonell y Pompeu 2000; Pérez et al. 2005).

Ante la respuesta positiva del rendimiento de fibra en Buenos Aires y Bella Cecilia, es probable que esto sea una consecuencia de un mayor nivel de radiación (datos no mostrados en el presente experimento); Raffo e Iglesias (2004), indican que la radiación fotosintéticamente activa captada por una planta determina la cantidad de materia seca producida y la calidad de la producción, influyendo sobre el crecimiento y la productividad (Hernández et al.,2016; Jarma 2006). Los factores ambientales tales como 
la radiación, temperaturas y humedad pueden afectar las expresiones fenotípicas y fisiológicas de las plantas (Taiz Zaiger 1998), ya que se afectan los procesos de fotosíntesis, transpiración, alargamiento celular, crecimiento y competencia entre especies (Mengel y Kirby 2001; jarma et al.,2008). Esto también podría explicar el comportamiento diferencial de los genotipos y los ambientes observados en este trabajo.

En la figura 2 se muestra las medias de los genotipos por localidad para Pn. En la localidad de Buenos Aires se registraron los mayores valores de $\mathrm{Pn}$ en los genotipos Criolla, Criolla 1, Criolla 2 y Martinera, con valores de 10,4; 11,$2 ; 12,2$ y $12,1 \mu \mathrm{mol} \mathrm{CO} 2 \mathrm{~m}^{-2} \mathrm{~s}^{-1}$ respectivamente (Figura 2a).

Para el caso de la localidad Instecavi los mayores valores de Pn se observaron en los genotipos Criolla, Criolla 1 presentándose el orden siguiente: Criolla $=$ Criolla $1>$ Criolla $2=$ Martinera $>$ Costanera, con valores respectivos de 9,4; 8,3; 7,$2 ; 6,5$ y $4,8 \mu \mathrm{mol} \mathrm{CO} \mathrm{Cm}^{-2} \mathrm{~s}^{-1}$ (Figura $2 \mathrm{~b}$ ).

En Bella Cecilia los mayores valores de $\mathrm{Pn}$ se registraron en los genotipos Criolla, Criolla 1 , Criolla 2, presentándose el siguiente orden Criolla $=$ Criolla $1=$ Criolla $2>$ Martinera $=$ Costanera, con valores de 13,4; 12,9; 12,8; 10,7 y $10,3 \mu \mathrm{mol}$ CO2 m-2 s-1 respectivamente (Figura 2c). En la localidad de Chimalito mayores valores de $\mathrm{Pn}$ se registraron en el genotipo Criolla, presentándose el siguiente orden CrioIla $>$ Criolla $1=$ Criolla $2=$ Martinera $>$ Costanera, con valores de 10,$9 ; 8,7 ; 9,3 ; 8,3$ y 7,0 $\mu \mathrm{mol} \mathrm{CO} \mathrm{Cm}^{-2} \mathrm{~s}^{-1}$ respectivamente (Figura $2 \mathrm{~d}$ ).

Para el caso de la localidad de La Oportunidad la prueba de comparación de medias indica que los mayores valores de $\mathrm{Pn}$ se registraron en los genotipos Criolla, Criolla 1, Criolla 2 y Martinera, con valores respectivos de 11,6;
10,2 y $10,1 \mu \mathrm{mol} \mathrm{CO}_{2} \mathrm{~m}^{-2} \mathrm{~s}^{-1}$ (Figura 2e). En la localidad de Bajo Grande los mayores valores de $\mathrm{Pn}$ se registraron en los genotipos Criolla, Criolla 1, Criolla 2 y Martinera, con valores de 7,$8 ; 8,4 ; 9,1$ y $9 \mu \mathrm{mol} \mathrm{CO}_{2} \mathrm{~m}^{-2} \mathrm{~s}^{-1}$ respectivamente (Figura $2 \mathrm{f}$ ).

En La Abisinia los mayores valores de Pn se registraron en los genotipos Criolla, Criolla 1, Criolla 2, presentándose el siguiente orden Criolla $=$ Criolla $1=$ Criolla $2>$ Martinera $=$ Costanera, con valores de 8,$5 ; 8,4 ; 8,3 ; 7$ y $6,7 \mu \mathrm{mol} \mathrm{CO}_{2} \mathrm{~m}^{-2} \mathrm{~s}^{-1}$ respectivamente (figura $2 \mathrm{~g}$ ). Por último, los mayores valores de Pn en la localidad de Tófeme se registraron en los genotipos Criolla, Criolla 1 presentándose el siguiente orden Criolla $=$ Criolla $1>$ Criolla 2 = Martinera > Costanera, con valores respectivos de 7,$1 ; 6,3 ; 5,4 ; 4,9$ y $3,6 \mu \mathrm{mol} \mathrm{CO} 2 \mathrm{~m}-2$ s-1 (Figura 2h).

\section{Conclusiones}

Se evidenciaron mayores rendimientos de fibra en los genotipos Criolla y Criolla 1 respecto al resto de genotipos, así como un efecto benéfico de las localidades Buenos Aires, Bella Cecilia y Chimalito. Se obtienen rendimientos de fibra más estables cuando se aplica fertilización orgánica tanto en localidades favorables como desfavorables. Las tasas de fotosíntesis de los genotipos Criolla y Criolla 1 fueron superiores en la mayoría de las localidades y las desventajas en la adaptación que presentarían genotipos como Martinera y Costanera. Los genotipos Martinera y Costanera fueron las menos adaptadas.

\section{Agradecimientos}

Este trabajo fue financiado por la Gobernación de Córdoba y la Universidad del Sinú como entidad cooperante, en la actividad "evaluación fisiológica de la adaptación de 5 genotipos de caña flecha (Gynerium saggittatum Aubl), en 
los resguardos indígenas de San Andrés de Sotavento y Tuchín, en el departamento de Córdoba, bajo dos (2) niveles de fertilización" en el marco del proyecto " Desarrollo e investigación aplicada de un modelo experimental sostenible e innovador en la cadena productiva de artesanías derivadas de la Caña Flecha (Gynerium saggittatum Aubl.) en el departamento de Córdoba BPIN: 2012000100179.

\section{Literatura Citada}

Aramendiz, H., Espitia, M. y Robles, J. (2005). Colección, conservación, caracterización morfoagronómica y producción de semilla de caña flecha (Gynerium sagitatum Aubl.) del Caribe Colombiano. CIUC. Universidad de Córdoba, Montería. $118 \mathrm{p}$.

Carbonell, S. y Pompeu, A. (2000). Estabilidade fenotípica de linhagens de feijoeiro em três épocas de plantio no estado de São Paulo. Pesquisa Agropecuria Brasileira 35(2):321 - 329. http:// dx.doi.org/10.1590/S0100-204X2000000200011

Carneiro PCS (1998) Novas metodologias de análise da adaptabilidade e estabilidade de comportamento. Tese de Doutorado. Universidade Federal de Viçosa, Viçosa. 155p.

Casas Caro, L. F., Arévalo Ramírez, C. D. P., Garavito Carvajal, C. P., \& Rodríguez Forero, G. M. (2010). Cartilla para la producción sostenible de artesanías en caña de flecha.

Durango, É. y Humanez, A. (2017). Enraizamiento de esquejes de Caña Agria (Cheilocostus speciosus. J. Koenig). Revista Colombiana de Biotecnología, 19(2), 133-139.

Hernández, J.L. Combatt, E.M. Jarma, A. Polo, J. Rodríguez, L. (2016). Rendimiento y calidad de hojas de Stevia rebaudiana Bert. bajo la oferta edafologica y dos niveles de radiación en cinco regiones de Colombia. Revista U.D.C.A Actualidad \& Divulgación Científica 19 (1): 77 - 85. ISSN 0123-4226.

Jarma, A. (2008). Estudios de adaptación y manejo integrado de estevia (Stevia rebaudiana Bert.): nueva alternativa agroindustrial del Caribe colombiano. Una revisión. Rev. Colomb. Cien.
Hort. 2(1):109 - 120. https://doi.org/10.17584/ rcch.2008v2i1.1176

Jarma, A. Rengifo, T. and Aramendiz, H. (2006). Fisiología de estevia (Stevia rebaudiana) en función de la radiación en el Caribe colombiano. II.: Análisis de crecimiento. Agron. colomb. [online]. 24(1): 38-47. ISSN 0120-9965.

Kang, M.S. (1998). Using genotype-by-environment interaction for crop cultivar evelopment. Advances in Agronomy, San Diego, v.62, p.199-252. https://doi.org/10.1016/S0065-2113(08)60569-6

Lin, C.S. and Binns, M.R. (1988). A superiority measure of cultivar performance for cultivar $x$ location data. Can. J. Plant Sci. 68: 193-198. https:// doi.org/10.4141/cjps88-018

López Rojas, S. L., Quiñones Rodríguez, N., \& Villada Camelo, J. (2014). Informe final del proyecto fortalecimiento de la labor productiva del oficio artesanal en caña flecha en el Resguardo Zenú de San Andrés de Sotavento.

Mekonnen, K. et al. (2006). Performance of eight tree species in the highland Vertisols of central Ethiopia: growth, foliage nutrient concentration and effect on soil chemical properties. New Forest, 32(3): 285-298. DOI 10.1007/s11056-006-9003-x

Mengel, K. y Kirby, E. (2001). Principles of plant nutrition. Kluwer Academic Publ., Dordrecht, The Netherlands.

PÉREZ, J.C. CEBALLOS, H. ORTEGA, E. LENIS, J. (2005). Análisis de la interacción genotipo por ambiente en yuca (Manihot esculenta Crantz) usando el modelo AMMI. Fitotecnia Colombiana. 5(2):11-19.

Raffo, M. y Iglesias, D. (2004). Efecto de la intercepción y distribución de la radiación fotosintéticamente activa en manzanos cv. 'Fuji', bajo cuatro sistemas de conducción en alta densidad. Revista de Investigaciones Agropecuarias (RIA) 33(2), 29-42.

Rivera, H.J. Suárez, I.E. Palacio, J.D. (2009). Análisis de la diversidad genética de la caña flecha Gynerium sagittatum Aubl. utlilizandola como tecnica de AFLP. Agric. Téc. Méx. 35 (1): 81-87. ISSN 0568-2517

Rodriguez-Gonzalez, R.E. et al. (2011). Interacción genotipo-ambiente para la estabilidad de rendimiento en trigo en la región de Mexicali, B.C., México. Trop. subtrop. agroecosyt [online]. vol.14, n.2, pp. 543-558. ISSN 1870-0462. 
Ruiz, J.A. Flores, H.E. Ramírez, J.L. y González, D.R. (2002). CARDINAL TEMPERATURES AND LENGTH OF MATURATION CYCLE OF MAIZE HYBRID H-311 UNDER RAINFED CONDITIONS. AGROCIENCIA. 36 (5), 569-577-

SNE-Servicio de Noticias del Estado. (2004). El sombrero vueltiao ahora es símbolo cultural de la Nación. En: Casa de Nariño, Presidencia de la República, http://www.presidencia.gov.co/ sne/2004/septiembre/10/02102004.html.

SOUZA, A.A. (2005). Interaction Genotype X Environment in cotton (Gossypium hirsutum L.r. latifolium Hutch) through the methods of adaptability, stability, and environmental grouping. Areia, PB: UFPB/CCA. 126p: il. Tese (Doctorate in Agronomy - Plant Breeding Genetic) - Centro de Ciências Agrárias, Universidade Federal da Paraíba.

Taiz, L. Zeiger, E. (2010). Plant physiology. Fifth edition, Sinauer Associates, Incorporated, Redwood City, CA, p. 644-645.

Taiz, L. y Zeiger, E. (1998). Plant physiology. Second edition. Sinaver Associates Inc. Publishers, Sunduerland, USA. pp. 228-229. 
